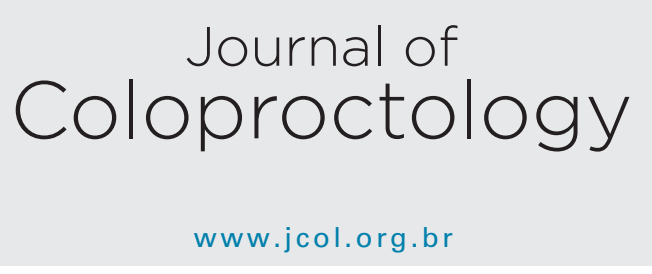

\title{
Editorial
}

\section{Taylored prevention of postoperative endoscopic recurrence in Crohn's disease: new data for an old problem}

\section{Prevenção individualizada de recorrência endoscópica pós-operatória na doença de Crohn: novos dados para um antigo problema}

Crohn's disease (CD) is a recurrent entity, and is not curable even with surgery. ${ }^{1}$ Despite the ultimate advances in its management, including new diagnostic tools (such as fecal calprotectin and enterography with magnetic resonance imaging), as well as efficacious therapeutic agents (such as biological drugs), surgery still plays a significant role in the CD treatment algorithms. ${ }^{1}$ Populational studies demonstrated that after 20 years of diagnosis, up to $80 \%$ of the patients may need at least one intestinal resection during the natural course of the disease. ${ }^{2}$ Recurrence of CD is common, and usually occurs near the anastomosis of the previous operation. ${ }^{3}$

There are several types of recurrence in CD. Histologic recurrence is often the first to occur, and can be observed weeks after an operation that included resection. Endoscopic recurrence usually occurs around one year after the surgical resections, and precedes clinical (symptomatic) and surgical recurrences. ${ }^{3}$ Therefore, postoperative endoscopic recurrence (PER) is one of the initial signs of future symptoms, future need for multiple operations and disability. The occurrence of PER and its possible causes had been studied for decades, since the pivotal study by Rutgeerts et al., in $1990 .^{4}$

Several strategies had been described in the prevention of PER, as well as its management once it is clearly detected. Stratification of the patients regarding their risk for recurrence, according to several risk factors such as smoking, previous resections, perforating $C D$ and mioenteric plexitis, can help physicians to define the best drug to be used in each individual case. ${ }^{3}$ Traditionally, patients with high risk for recurrence (with one or more of the risk factors previously stated) are managed with more powerful drugs, such as immunomodulators and TNF-alpha inhibitors, in order to reduce PER rates. Patients with low risk for recurrence, usually those with the first resection and luminal disease, can be managed with antibiotics and close follow-up, even without any treatment for some periods. ${ }^{3}$

The difficulties that we face as physicians, are mostly based on the uncertain course of the disease after an operation. We must avoid overtreatment (treating patients with biological agents without a clear need for this therapy), due to the risks of adverse events, as well as important cost-efficacy issues. On the other hand, we must also avoid undertreatment, which means to ignore risk factors and simply treat patients with worse prognostic factors with drugs of uncertain efficacy. In summary, we cannot treat all patients with a single strategy, and data regarding comparison of different algorithms are scarce.

In February 2014, during the European Crohn's and Colitis (ECCO) congress, in Denmark, the final results of the POCER trial were presented..$^{5}$ This was the first prospective multicenter trial that compared the rates of PER after two different strategies of prevention of its occurrence. This study included patients with "curative" ileocecal resection, without any residual $C D$, that were followed for 18 months. In one arm of the study, patients were treated according to their risk factors in isolation, with the best drug regimen at hand, and had a colonoscopy after 18 months to check the rates of PER. In the other arm, patients were initially treated according to their risk factors, but a colonoscopy after 6 months of the resection could define the patients already with PER, and escalate them to the next therapeutic agent, in order to optimize PER reduction at the end of the 18 months period of the study. In summary, the treat-to-target strategy (aiming mucosal healing, with an interventional colonoscopy) was tested against regular treatment. 
The results of the POCER trial (with 174 patients) demonstrated that patients who had their therapeutic decision based on a colonoscopy 6 months after the operation (active care arm), had lower PER rates (49\%) after 18 months than patients that were treated according to the best drug regimen $(67 \%$ standard care arm), with $\mathrm{p}=0.028 .^{5}$

The conclusions of this important trial, with a high level of evidence, were that treating according to the risk of recurrence, with early colonoscopy and treatment step-up for recurrence, was superior to optimal drug therapy alone in preventing post-operative CD recurrence. Another important lesson from this study, was that selective immunosuppression (with azathioprine and Adalimumab, when needed), with colonoscopy-based adjustment, rather than its use in all high risk patients, lead to effective disease control in the majority of patients. Tayloring the treatment demonstrated better results than treating every patient with a fixed strategy.

In an era where biological agents are fully available worldwide, the selection of the proper use of these drugs for the proper patients is essential to avoid overtreatment. Therefore, the aphorism that every patient submitted to an operation needs to escalate therapy to the biological level, is not fully accepted. Although not yet clear in the majority of Inflammatory Bowel Diseases guidelines, the strategy of a colonoscopy 6 months after an ileocecal resection to detect PER seems to be the best strategy to manage CD patients after surgery to date. Fecal calprotectin trials are also needed in order to optimize the timing for the first colonoscopy after surgery. Endoscopic assessment seems to be essential for a more precise definition of the best drug for our patients. The POCER trial demonstrated the best evidence in the management of PER in the last years. It brought good and new data to face the old problem of recurrence.

As we know, CD is a lifelong disease. Therefore, studies with longer follow-up periods are still needed, to check the benefits of the strategy demonstrated in the POCER trial in the long term. There are still lots of questions to be determined in the management of PER. We hope that this old problem can have better and new solutions in the years to come.

\section{REFERENCES}

1. Baumgart DC, Sandborn WJ. Crohn's disease. Lancet. 2012;380(9853), 1590-605.2.

2. Munkholm P, Langholz E, Davidsen M, Binder V. Intestinal cancer risk and mortality in patients with Crohn's disease. Gastroenterology. 1993;105(6):1716-23.

3. De Cruz P, Kamm MA, Prideaux L, Allen PB, Desmond PV. Postoperative recurrent luminal Crohn's disease: a systematic review. Inflamm Bowel Dis. 2012;18(4):758-77.

4. Rutgeerts P, Geboes K, Vantrappen G, Beyls J, Kerremans R, Hiele M. Predictability of the postoperative course of Crohn's disease. Gastroenterology. 1990;99(4):956-63.

5. Kamm MA, De Cruz PP, Wright EK, Hamilton AL, Ritchie KJ, Krejany EO, et al. Optimising postoperative Crohn's disease management: best drug therapy alone versus endoscopic monitoring, disease evolution and fecal calprotectin monitoring. The POCER study. OP 23 at the ECCO congress, 2014, Copenhagen, Denmark. $<$ https://www.ecco-ibd.eu/publications/congress-abstracts/abstracts-2014/item/op023-optimising-post-operative-crohns-disease-management-best-drug-therapy-alone-versusendoscopic-monitoring-disease-evolution-and-faecalcalprotectin-monitoring-the-pocer-study.html>. Accessed in March 22nd, 2014

Paulo Gustavo Kotze CO-editor of JCOL

E-mail: pgkotze@hotmail.com

http://dx.doi.org/10.1016/j.jcol.2014.04.003 2237-9363/@ 2014 Sociedade Brasileira de Coloproctologia. Published by Elsevier Editora Ltda. Este é um artigo Open Access sob a licença de CC BY-NC-ND 